\title{
Neural Substrates for Head Movements in Humans: A Functional Magnetic Resonance Imaging Study
}

\author{
Cecilia N. Prudente, ${ }^{1}$ Randall Stilla, ${ }^{1}$ Cathrin M. Buetefisch, ${ }^{5}$ Shivangi Singh, ${ }^{1}$ Ellen J. Hess, ${ }^{2}$ Xiaoping Hu, ${ }^{6}$ \\ Krish Sathian, ${ }^{3,7}$ and H.A. Jinnah ${ }^{4}$ \\ ${ }^{1}$ Department of Neurology, ${ }^{2}$ Departments of Pharmacology and Neurology, ${ }^{3}$ Departments of Neurology, Rehabilitation Medicine, and Psychology, and \\ ${ }^{4}$ Departments of Neurology, Human Genetics, and Pediatrics, Emory University, Atlanta, Georgia, 30332, 5Departments of Neurology and Rehabilitation \\ Medicine, Emory University, Atlanta, Georgia 30309, ${ }^{6}$ Coulter Department of Biomedical Engineering, Emory University and Georgia Institute of \\ Technology, Atlanta, Georgia 30322, and 7Rehabilitation R\&D Center of Excellence, Atlanta VA Medical Center, Decatur, Georgia 30033
}

The neural systems controlling head movements are not well delineated in humans. It is not clear whether the ipsilateral or contralateral primary motor cortex is involved in turning the head right or left. Furthermore, the exact location of the neck motor area in the somatotopic organization of the motor homunculus is still debated and evidence for contributions from other brain regions in humans is scarce. Because currently available neuroimaging methods are not generally suitable for mapping brain activation patterns during head movements, we conducted fMRI scans during isometric tasks of the head. During isometric tasks, muscle contractions occur without an actual movement and they have been used to delineate patterns of brain activity related to movements of other body parts such as the hands. Healthy individuals were scanned during isometric head rotation or wrist extension. Isometric wrist extension was examined as a positive control and to establish the relative locations of head and hand regions in the motor cortex. Electromyographic recordings of neck and hand muscles during scanning ensured compliance with the tasks. Increased brain activity during isometric head rotation was observed bilaterally in the precentral gyrus, both medial and lateral to the hand area, as well the supplementary motor area, insula, putamen, and cerebellum. These findings clarify the location of the neck region in the motor homunculus and help to reconcile some of the conflicting results obtained in earlier studies.

Key words: fMRI; head movements; isometric; motor control; neck; somatotopic

\section{Introduction}

Head movements in humans are highly complex. At the most basic level, the head must be supported against the force of gravity. Head movements also orient sensory structures of the head, especially those for vision, with both slow tracking movements and rapid redirections. Further, head movements such as nodding or shaking are used for nonverbal communication. These different types of movements are likely to require distinct control mechanisms and several different neural pathways.

Animal studies have suggested that specific regions in the cerebral cortex, basal ganglia, cerebellum, and brainstem are in-

\footnotetext{
Received March 1, 2015; revised April 21, 2015; accepted May 10, 2015.

Author contributions: C.N.P., C.M.B., E.J.H., X.H., K.S., and H.A.J. designed research; C.N.P., R.S., S.S., and H.A.J. performed research; C.M.B. contributed unpublished reagents/analytic tools; C.N.P., R.S., C.M.B., S.S., E.J.H., X.H., K.S., and H.A.J. analyzed data; C.N.P., K.S., and H.A.J. wrote the paper.

This work was supported the National Institute of Neurological Disorders and Stroke (Grants U54NS065701 and U54NS06571-03S1) and the Office of Rare Diseases Research in the National Center for Advancing Translational Sciences at the National Institutes of Health; the Emory University Research Committee (Grant UL1 RR025008), and the Pilot Projects Program from the Emory Biomedical Imaging Technology Center. K.S. is supported by the Veterans Administration. We thank all volunteers for participating in the study; Teresa Kimberley for assistance with data analysis and interpretation; and Doug Bernhard, Simon Lacey, Laura Rose, and Ami Rosen for technical assistance and help with subject recruitment.

The authors declare no competing financial interests.

Correspondence should be addressed to H.A. Jinnah, MD, PhD, 6300 Woodruff Memorial Research Building, 101 Woodruff Circle, Department of Neurology, Emory University, Atlanta, GA 30322. E-mail: hjinnah@emory.edu. DOI:10.1523/JNEUROSCI.0851-15.2015

Copyright $\odot 2015$ the authors $\quad 0270-6474 / 15 / 359163-10 \$ 15.00 / 0$
}

volved in the control of head movements (Isa and Sasaki, 2002; Peterson, 2004). However, how these different regions contribute to head movements in humans remains largely unexplored. Regarding the primary motor cortex (M1), two fundamental questions remain unanswered. First, it is still debated whether neck muscles are controlled ipsilaterally, contralaterally, or bilaterally (Table 1). Second, the exact location of the neck motor region in the somatotopic organization of M1 is not clear (Fig. 1). Classic experiments that mapped M1 by direct electrical stimulation suggested that the neck region is represented laterally on the convexity of the cerebral hemisphere between the finger and face areas (Rasmussen and Penfield, 1948; Penfield and Rasmussen, 1950). In contrast, other studies suggested that the neck region is located more medially, between the representations of the trunk and arm (Obrador, 1953; Thompson et al., 1997; Kang et al., 2011; Pirio Richardson, 2014), in parallel with the representation of the neck in the somatosensory homunculus (Penfield and Rasmussen, 1950).

There are several reasons for the lack of conclusive information on the neural control of head movements in humans. Electrophysiological mapping studies typically use a fixed frame to restrain the head during testing and neck muscles often show inconsistent responses in transcranial magnetic stimulation studies (Hanajima et al., 1998; Pirio Richardson, 2014). Furthermore, functional neuroimaging methods are problematic because head motion during scanning degrades data quality. We have ad- 
Table 1. Prior reports of the hemisphere controlling head movements in humans

\begin{tabular}{|c|c|c|}
\hline Ipsilateral & Contralateral & Bilateral \\
\hline Beevor (1909) ${ }^{a}$ & Hanajima et al. (1998) ${ }^{b}$ & Penfield and Rasmussen $(1950)^{b}$ \\
\hline Balagura and Katz (1980) ${ }^{a}$ & Kang et al. (2011) ${ }^{a}$ & Benecke et al. (1988) ${ }^{b}$ \\
\hline Willoughby and Anderson & & Gandevia and Applegate (1988) ${ }^{b}$ \\
\hline$(1984)^{a}$ & & Berardelli et al. (1991) \\
\hline Mastaglia et al. (1986) ${ }^{a}$ & & Odergren and Rimpilainen (1996) \\
\hline Manon-Espaillat and Ruff & & Odergren et al. (1997) ${ }^{b}$ \\
\hline$(1988)^{a}$ & & Thompson et al. (1997) ${ }^{b}$ \\
\hline Anagnostou et al. (2011) ${ }^{a}$ & & DeToledo and Dow (1998) ${ }^{a}$ \\
\hline
\end{tabular}

The hemisphere controlling movements was taken from the results reported by each study, because the interpretations sometimes did not match the actual results provided.

astudies of stroke or epilepsy cases.

${ }^{b}$ Studies of electrical or magnetic stimulation of the precentral gyrus.

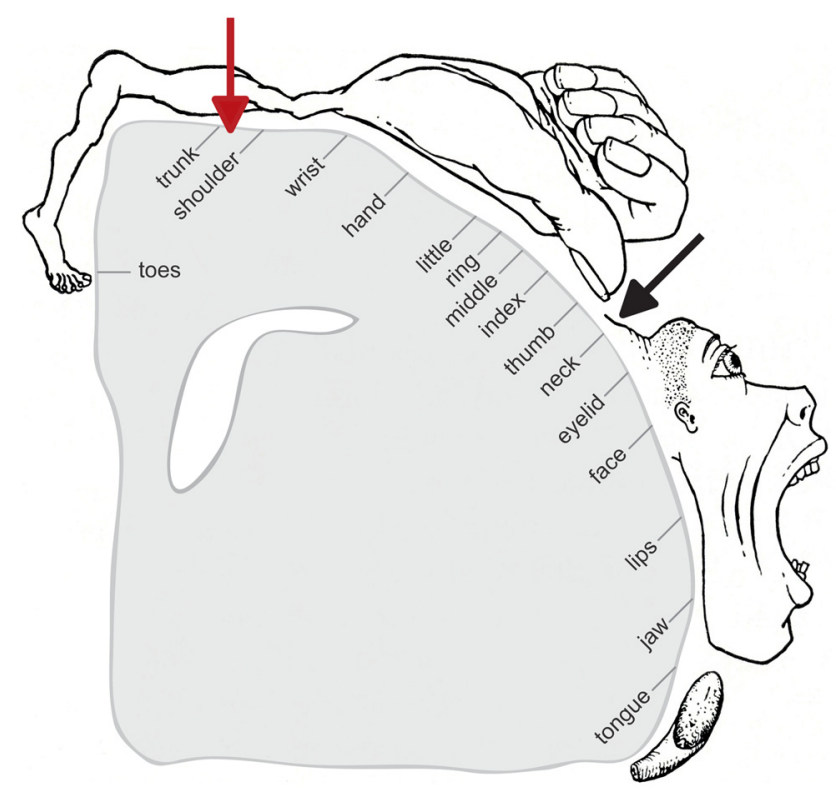

Figure 1. Representation of the neck area in the motor homunculus among different studies. The black arrow indicates the neck area identified by Penfield and Rasmussen (1950). The red arrow indicates the neck area identified in other studies (Thompson et al., 1997; Kang et al., 2011; Pirio Richardson, 2014). This figure was adapted from Penfield and Rasmussen (1950).

dressed these obstacles by using an isometric head rotation task during fMRI to examine the neural substrates of head control in humans. In isometric tasks, muscles are activated without actual movements. Prior studies have shown that isometric hand movements activate similar brain regions as actual hand movements (van Duinen et al., 2008; Keisker et al., 2010), providing a strategy for addressing the usual technical limitations of assessing head movements during fMRI.

\section{Materials and Methods}

Participants. All procedures were approved by the Emory University Institutional Review Board and all participants gave informed consent. All subjects were neurologically normal and had the ability to perform a full range of head movements in all directions. Participants were excluded if they had significant orthopedic problems affecting the cervical spine, difficulty lying supine, abnormal head movements at rest, significant neck pain, contraindications for MRI, or untreated psychiatric problems. Eighteen participants were scanned, but all data from one were excluded due to excessive motion (see below). Therefore, data from 17 participants ( 12 women, 5 men) were included in the final analyses. Their mean age was $56.8 \pm 14.5$ years (range, $30-74$ ), 14 were right-handed, and three were left-handed.
MR scanning. Scans were performed on a 3 tesla Siemens Trio TIM scanner using a quadrature transmit-receive head coil at the Emory Biomedical Imaging Technology Center. Total scanning time was $\sim 25 \mathrm{~min}$. Functional images with blood oxygenation level dependent (BOLD) contrast were acquired using a $\mathrm{T} 2{ }^{*}$-weighted single-shot gradient-recalled echoplanar imaging sequence with the following parameters: 30 axial slices of $4 \mathrm{~mm}$ thickness, repetition time (TR) $2040 \mathrm{~ms}$, echo time (TE) 30 $\mathrm{ms}$, flip angle (FA) $90^{\circ}$, in-plane resolution $3.4 \times 3.4 \mathrm{~mm}^{2}$, and in-plane matrix $64 \times 64$. After the functional imaging runs, a 3D T1-weighted sequence (MPRAGE) of 176 sagittal slices of $1 \mathrm{~mm}$ thickness was obtained with TR $2300 \mathrm{~ms}$, TE $3 \mathrm{~ms}$, inversion time $1100 \mathrm{~ms}$, FA $8^{\circ}$, in-plane resolution $1 \times 1 \mathrm{~mm}^{2}$, and in-plane matrix $256 \times 256$. Acoustic noise attenuation was provided by headphones (Etymotic Research) that were also used to convey instructions and audio cues for each task.

Experimental design. Scanning was conducted during isometric head or hand tasks. All tasks were practiced outside the scanner first. Head tasks consisted of submaximal isometric horizontal head rotation to the right or left. Actual head movements during tasks were prevented by firm foam padding around the head and restraining straps within the head coil placed tightly across the forehead and chin. To avoid eye movements, subjects were asked to look at all times at a white cross projected on a black screen viewed via a mirror mounted inside the head coil.

Hand tasks were investigated as a positive control because they reliably give robust signals and because their cortical representations have been more extensively mapped than other body regions closer to the neck, such as the shoulder or lower face. Hand tasks consisted of submaximal isometric wrist extension of either hand, the arm being positioned in a neutral position between pronation and supination. Therefore, right wrist extension was associated with a rightward movement effort and left wrist extension with a leftward movement effort, matching the corresponding directions for head rotation. Sandbags placed along the dorsal and lateral aspects of both arms and hands prevented actual hand movements.

Functional data were collected during two runs. A block design was used with alternating blocks of active tasks and rest periods (Fig. 2). Each run consisted of 16 active blocks (four per condition), separated by rest periods of $12.24 \mathrm{~s}$; a rest period also occurred at the start and end of each run. The task conditions were as follows: isometric head rotation to the right, isometric head rotation to the left, isometric right wrist extension, and isometric left wrist extension. Task blocks were interleaved in a predetermined pseudorandom sequence. Each active block lasted $20.4 \mathrm{~s}$ and involved 4 repetitions of a single task separated by periods of $1 \mathrm{~s}$.

The timing of stimulus presentations was provided by audio cues controlled by Presentation software (Neurobehavioral Systems). Immediately preceding each block, an audio cue was presented for $1 \mathrm{~s}$ to prepare the subject for the next condition. The following cues were used: "relax," "press right cheek," "press left cheek," "press right wrist," or "press left wrist." Empirically, these cues were found in pilot studies to lead to fewer head movements compared with instructions to "rotate" or "turn" the head. Timing for each movement during task blocks was cued by beeps played at a frequency of $0.2 \mathrm{~Hz}$.

Electromyography. Because the presence or absence of actual movements could not be verified visually during scanning, compliance with isometric tasks during practice and scanning was confirmed by surface electromyography (EMG) of the sternocleidomastoid and the extensor carpi ulnaris muscles bilaterally. The sternocleidomastoid is an agonist in contralateral horizontal rotation of the head and it is active during contralateral isometric head rotation (Vitti et al., 1973). The extensor carpi ulnaris is involved in wrist extension and is active in ipsilateral isometric wrist extension (Divekar and John, 2013). Pilot studies confirmed that these same muscles were activated reliably during the isometric tasks investigated.

EMG procedures, electrode placement, and safety guidelines followed published protocols (van Duinen et al., 2008; Criswell and Cram, 2010; Nöth et al., 2012). The safety guidelines dictated the use of a transmitreceive head coil (see above) rather than other available coils permitting higher-resolution, parallel imaging. EMG recordings were collected using MRI-compatible electrodes and Brain Vision Recorder version 1.20 (Brain Products) with a sampling rate of $5000 \mathrm{~Hz}$. Data processing was completed with Brain Vision Analyzer version 2.0 (Brain Products) and 


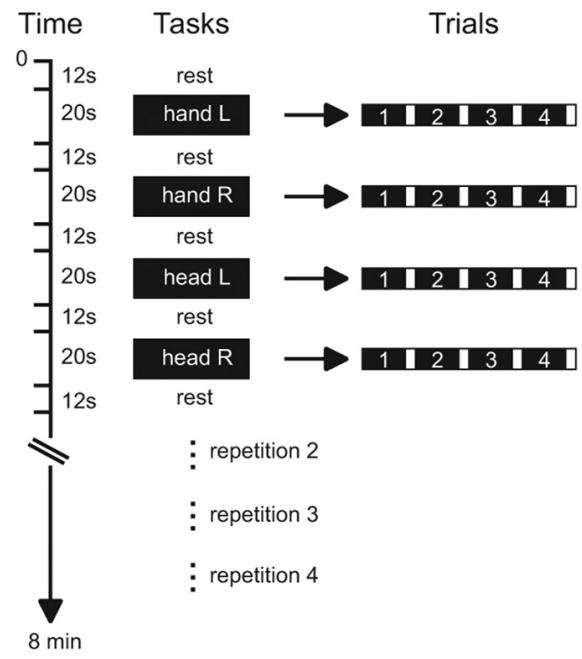

Figure 2. Experimental design. Tasks consisted of isometrichead rotations to the right or left and isometric right or left wrist extensions. Each active block consisted of four trials of the same task. The sequence of active tasks blocks was pseudo-randomly repeated four times within each run.

involved removal of imaging-related artifacts, signal filtering with low cutoff frequency of $20 \mathrm{~Hz}$, and signal rectification.

EMG recordings were verified during practice and during and after scanning. During practice sessions and before scanning, we first verified that EMG signals corresponded to activation of the muscles expected for each type of isometric or actual movement for all cases. After imaging was completed, all EMG recordings were evaluated by an observer blinded to tasks to verify activation of the correct muscles for each task. Active and rest periods were defined based on the relative level of muscle activation compared with background activity.

Head motion correction. Several measures were taken to ensure that head motion during scanning was minimized. These included practice periods before scans, stabilization of the head with firm supports in the scanner, and motion correction of imaging data.

Motion correction of imaging data was completed in two stages to account for head motion during scanning. The first motion correction step occurred during data acquisition using the scanner's inbuilt software Prospective Acquisition Correction (3D-PACE; Thesen et al., 2000). 3DPACE allows detection and correction of head motion during data acquisition and is able to compensate for translation (displacement) and rotation in the $x, y$, and $z$ planes within a single scanning run. With $3 \mathrm{D}-\mathrm{PACE}$, each brain volume is compared with the previous one and head motion is calculated and displayed in real time. For acquisition of the next dataset, slice position and orientation are adjusted according to the altered position of the head. Therefore, the correction of motion is done prospectively on the actual imaging data acquired for every TR.

The second motion correction step involved the alignment of brain volumes using sinc interpolation in BrainVoyager (Brain Innovation) to account for head movements that occurred between different runs. When multiple runs of a task are collected during a scanning session, 3D-PACE corrects for movement within each run. However, subjects may move between runs. To compensate for this potential betweenrun movement, an intrasession alignment of all brain volumes is beneficial. For this alignment, a target brain volume was selected from the functional run collected closest in time to the structural scan because images collected during this run had the smallest variance in space with images from the structural scan. All other brain volumes within the scanning session were aligned to this target volume in BrainVoyager using an algorithm that matches structure better than the algorithm used by $3 \mathrm{D}$ PACE. This intrasession alignment of all brain volumes over a scanning session produces the most precise alignments.

Head motion analyses. In addition to the motion correction of imaging data, we also examined actual head motion during scanning. This analysis determined how much motion correction was required by $3 \mathrm{D}-\mathrm{PACE}$ because the software does not store a log file with the movement parameters required for correction. We examined the "raw" data (not corrected by $3 \mathrm{D}-\mathrm{PACE}$ ) in BrainVoyager, which generated motion log files with the six motion parameters representing the amplitude of head movement that actually occurred during scanning. These motion log files from BrainVoyager were exported to MATLAB R2014a (version 8.3.0.532; The MathWorks) and analyzed with custom scripts. In MATLAB, rotation values were transformed from degrees to millimeters using each subject's brain dimensions for the transformation. Next, all data were evaluated for movement amplitude in each plane. Additional analyses included searching for trends in head movement direction and task-related head motion.

In keeping with prior recommendations for acceptable head motion during fMRI scanning, we used a threshold of $1.75 \mathrm{~mm}$ (approximately half the size of a functional voxel) as the maximum head motion allowed in any plane (Poldrack et al., 2011). Therefore, if head motion was $>1.75$ $\mathrm{mm}$ either during an active task period or during rest, we selected a continuous sequence of blocks including the instant with excessive head movement and beginning and ending with a rest period. This continuous sequence of blocks was then removed from the final data analysis. We attempted to balance block numbers for each task within a run to minimize potential contributions of unbalanced trials numbers on activation magnitude maps. Using these criteria, all data for one subject were excluded because substantial portions of the data showed excessive head movement. For three other subjects, data for one to three blocks of active and rest periods were excluded because of excessive head motion.

Imaging data analysis. Image processing and analysis was performed using BrainVoyager QX 2.8.4 (Goebel et al., 2006). Individual functional data were preprocessed using cubic spline interpolation for slice scan time correction, sinc interpolation for intrasession alignment of functional volumes (as described above), and high-pass temporal filtering to two cycles per run to remove slow drifts in the data. Anatomic 3D images were processed, coregistered with the functional data, and transformed into Talairach space (Talairach and Tournoux, 1988). For group analyses, the data were spatially smoothed with an isotropic Gaussian kernel (full-width half-maximum $4 \mathrm{~mm}$ ) (White et al., 2001) and normalized across runs and subjects with the percent signal change transformation. BOLD signal time courses were obtained by averaging individual data points across blocks of the same type and then averaging across participants.

We used a two-stage strategy to delineate brain regions involved in the control of head movements. Because our primary aim was to identify the hemisphere and subregion of $\mathrm{M} 1$ associated with isometric head rotation to the right or left, the first stage was a region of interest (ROI) approach focused on the precentral gyrus, which includes M1. The second stage was an exploratory whole-brain analysis to investigate which regions other than the precentral gyrus were active during isometric head rotation. Statistical analyses for both approaches used the general linear model (GLM) method to model the hemodynamic response during active blocks compared with baseline, followed by group-level analysis treating participant as a random variable. Group activations during isometric tasks to the right or left were contrasted with the resting condition using Student's $t$ test on a voxel-by-voxel basis with a voxelwise significance level of $p<0.05$, corrected for multiple comparisons with a $3 \mathrm{D}$ extension of the clustercorrection method (Cluster Threshold Estimator plugin in BrainVoyager; Forman et al., 1995). Specifically for the ROI approach, the bilateral precentral gyrus was used as a mask in the GLM analysis. The mask was traced on the Talairach-normalized, aligned, and averaged brain from actual study participants $(n=17)$. This mask was defined using known anatomical landmarks (central sulcus, precentral sulcus, longitudinal fissure, cingulate sulcus, and lateral sulcus) to identify specific boundaries.

Group results for the ROI and whole-brain analyses were displayed on an "averaged anatomical brain" created in BrainVoyager by first selecting a representative (target) Talairach-normalized brain from the 17 subjects scanned. We then aligned the 16 remaining participants' Talairachnormalized brains individually to the target brain (coregistration to match gyral/sulcal pattern, followed by sinc transformation). The 16 transformed brains were then averaged and combined with the single 


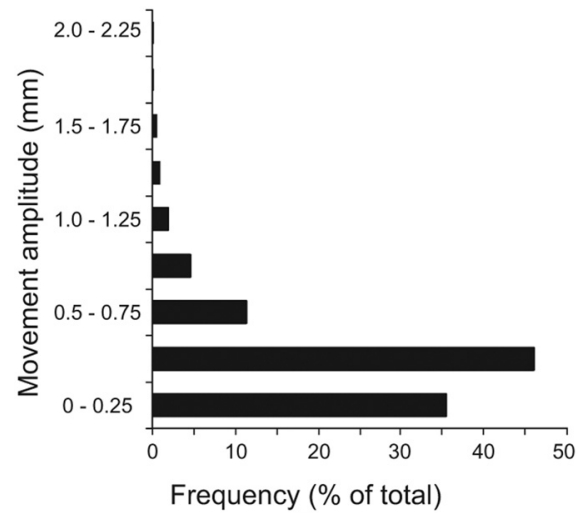

Figure 3. Distribution of head motion measurements and their amplitudes for all subjects. The $y$-axis represents the amplitude of each movement measured in millimeters. The $x$-axis shows the distribution of head motion measurements as a percentage of total values generated ( $n=17$ subjects; total data points: 49,788). Measurements for translational and rotational movements were combined for each plane of motion.

target brain, creating a Talairach template that was used to display the activations for the 17-participant group. Compared with standardized templates, this template more accurately reflects the specific anatomy of the actual subjects scanned. Activations were localized with respect to 3D anatomy with the help of MRI atlases (Duvernoy, 1999; Schmahmann et al., 1999; Cho, 2010).

This investigation included both sexes and either right-handed or lefthanded individuals. Considering that handedness may affect brain activation maps (Adamo et al., 2012), we examined the fMRI data for all right-handed $(n=14)$ and left-handed $(n=3)$ participants separately. No major differences were observed when considering tasks compared with rest for right-handed group alone or when comparing the righthanded group with the entire group. Similarly, considering that sex may affect neural structure and function (Sacher et al., 2013), we also examined the fMRI data for females $(n=12)$ and males $(n=5)$ separately. Once again, no major differences were observed for individual sexes compared with the whole group. Although these analyses cannot rule out subtle effects of handedness or sex, our plan was not designed to address these variables and the results presented here represent the findings for all 17 participants combined regardless of handedness or sex.

\section{Results}

\section{Task confirmation}

Participants were able to complete all tasks adequately as judged by direct observations during training and EMG during scanning. The expected muscles for each task were activated correctly for $99.0 \%$ and $97.1 \%$ of all head rotation trials for the right and left sternocleidomastoids, respectively, and for $97.1 \%$ and $94.2 \%$ of all wrist extension trials for the right and left extensor carpi ulnaris, respectively.

\section{Head motion during scans}

To verify that isometric head rotation was not associated with significant head movements, head movements were examined in all three planes. For each participant, the analysis generated one measurement for each of six movement parameters (translation or rotation in three planes) for every TR analyzed, yielding 53,244 data points for the whole group. After exclusion of data with excessive motion as described in Materials and Methods, the final analysis was completed with a total of 49,788 data points. Figure 3 shows the distribution of these remaining data points and their amplitudes. The vast majority of head motion (99.9\%) was below the standard cutoff of $1.75 \mathrm{~mm}$, approximately half a voxel. Head movements were also examined for all three planes of translation

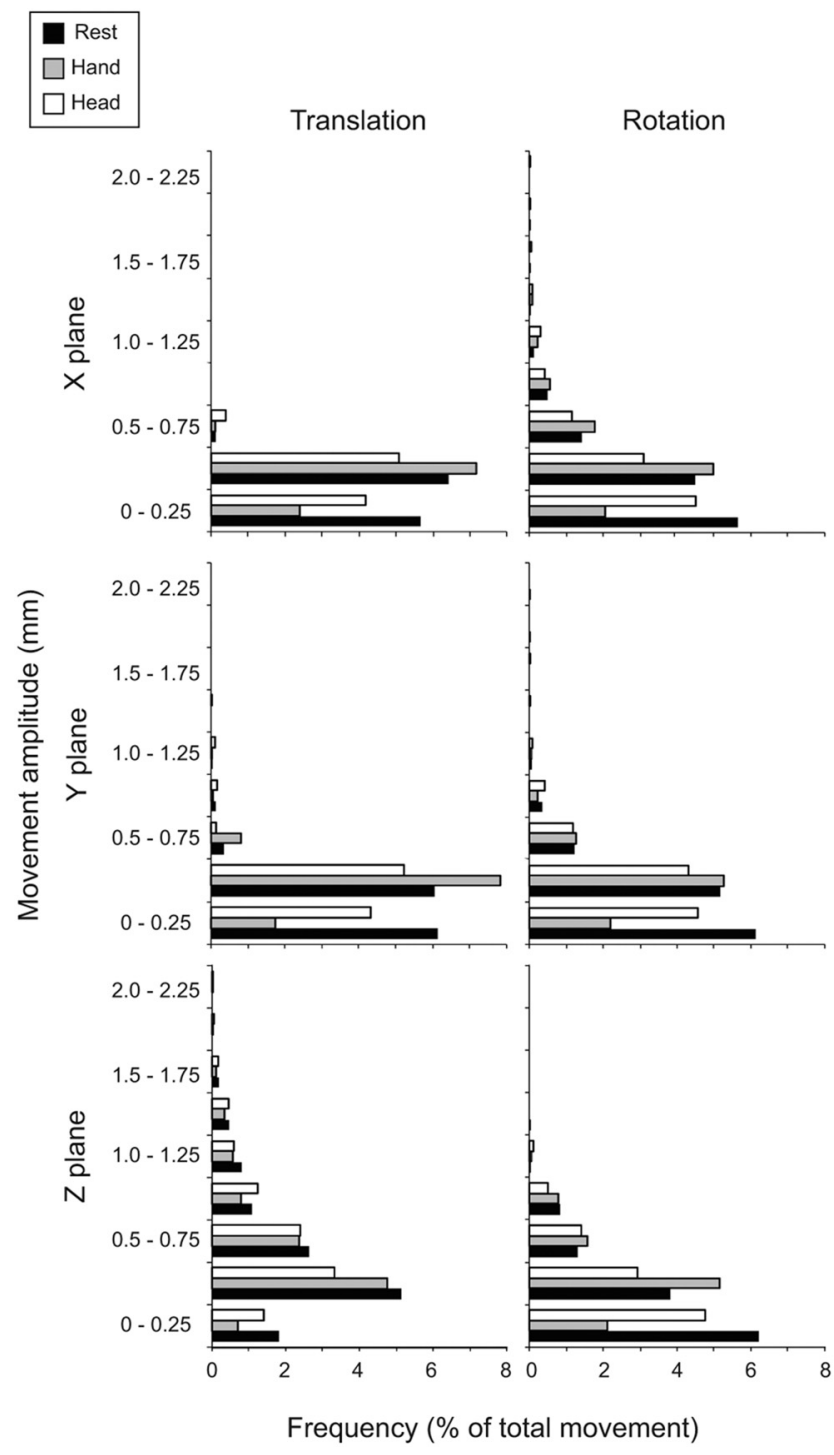

Figure 4. Head movements during rest, hand, and head tasks. The $y$-axis represents the amplitude of each movement measured in millimeters. The $x$-axis shows the distribution of head motion measurements as a percentage of total values generated ( $n=17$ subjects, total data points: rest: 9654; hand tasks: 7620; head tasks: 7620). Translational and rotational movements are shown for all three planes separately $(x, y$, and $z)$.

and rotation during rest and each type of task. The distributions of these movements showed that tasks and rest periods were associated with comparable movement during scanning (Fig. 4, Table 2). These results confirm that the imaging data were collected with minimal head movement, even for isometric head tasks.

\section{ROI analysis of hand tasks}

Isometric hand tasks were evaluated to provide a positive control and brain activation landmark for comparison with head tasks. Activation maps, Talairach coordinates, and BOLD signal curves for hand tasks compared with baseline are shown in Figure 5 and Table 3. Consistent with prior studies, isometric wrist extension with the right or left hand showed significant activation of the contralateral precentral gyrus in the area known as the "hand knob" (Yousry et al., 1997). For isometric right wrist extension, significant activation also was observed in a precentral area located more laterally and ventrally to the hand knob. These results 
Table 2. Head motion during scans

\begin{tabular}{|c|c|c|c|c|}
\hline Movement & Task & Plane & Mean & SD \\
\hline \multirow[t]{9}{*}{ Translation (mm) } & \multirow[t]{3}{*}{ Rest } & $x$ & 0.08 & 0.07 \\
\hline & & $y$ & 0.11 & 0.12 \\
\hline & & $Z$ & 0.33 & 0.31 \\
\hline & \multirow[t]{3}{*}{ Hand } & $x$ & 0.08 & 0.06 \\
\hline & & $y$ & 0.11 & 0.11 \\
\hline & & $z$ & 0.32 & 0.30 \\
\hline & \multirow[t]{3}{*}{ Head } & $x$ & 0.10 & 0.08 \\
\hline & & $y$ & 0.13 & 0.14 \\
\hline & & $Z$ & 0.37 & 0.33 \\
\hline \multirow[t]{9}{*}{ Rotation (mm) } & \multirow[t]{3}{*}{ Rest } & $x$ & 0.28 & 0.26 \\
\hline & & $y$ & 0.16 & 0.17 \\
\hline & & $Z$ & 0.21 & 0.17 \\
\hline & \multirow[t]{3}{*}{ Hand } & $x$ & 0.26 & 0.23 \\
\hline & & $y$ & 0.15 & 0.14 \\
\hline & & $Z$ & 0.21 & 0.16 \\
\hline & \multirow[t]{3}{*}{ Head } & $x$ & 0.31 & 0.31 \\
\hline & & $y$ & 0.19 & 0.17 \\
\hline & & $Z$ & 0.24 & 0.18 \\
\hline
\end{tabular}

confirm that isometric hand tasks produced patterns of activation similar to actual hand tasks.

\section{ROI analysis of head tasks}

Activation maps, Talairach coordinates, and BOLD signal curves for head tasks compared with baseline are shown in Figure 5 and Table 3. Isometric head rotation to the right showed significant activation of two foci in the contralateral precentral gyrus: one was medial and anterior to the hand knob and the other was lateral and ventral to the hand knob. Isometric head rotation to the left showed significant activation of bilateral precentral gyrus with two foci in each hemisphere: one located medial and anterior to the hand knob and the other lateral and ventral to the hand knob. For head rotation to either side, the locations of the medial precentral foci in each hemisphere appeared symmetrical, but the location of the lateral foci did not exactly match between hemispheres (Table 3).

The BOLD signal time courses for each condition (Fig. 5) showed that activation in the left lateral precentral focus was not specific to body region during rightward tasks. In contrast, there appeared to be greater selectivity (albeit relative) for the head in both left and right lateral precentral foci during leftward tasks. BOLD signal time courses for the left medial precentral focus showed low, nonselective activity during rightward tasks, but was more head-specific for leftward tasks, whereas the right medial precentral focus was nonselectively active for leftward tasks. The differences between isometric head rotation to the right or left may be due to actual hemispheric differences in right versus left head tasks, similar to differences previously reported for right and left hands (Triggs et al., 1999; Adamo et al., 2012). Alternatively, these differences may reflect statistical threshold effects because relaxing statistical stringency by evaluating clusteruncorrected maps showed bilateral activations of both medial and lateral precentral regions for both right and left head tasks.

\section{Whole-brain analysis of hand tasks}

Maps and Talairach coordinates of activations for hand tasks compared with baseline are shown in Figure 6 and Table 4. Isometric right wrist extension evoked contralateral activation in the hand knob, supplementary motor area (SMA), dorsal premotor area (PMd), ventral premotor area (PMv), primary somatosensory cortex (S1), parietal and frontal operculum, and putamen.
There also was significant activation of the ipsilateral cerebellar hemisphere in lobules IV and V.

Isometric left wrist extension activated the contralateral hand knob, bilateral SMA, contralateral S1, and parietal operculum. There was significant activation of the ipsilateral cerebellum, again mainly in lobules IV and V. These results are consistent with prior studies of both isometric and actual hand movements (Picard and Strick, 2001; Gerardin et al., 2003; van Duinen et al., 2008; Keisker et al., 2010; Mottolese et al., 2013).

\section{Whole-brain analysis of head tasks}

Maps and Talairach coordinates of activations for head tasks compared with baseline are shown in Figure 6 and Table 5. Isometric head rotation to the right activated the contralateral precentral gyrus, bilateral SMA, anterior insula, frontal operculum, and posterior putamen. Ipsilateral activation was observed in the mid-insula, anterior putamen, globus pallidus, and ventrolateral thalamus. No significant activation was observed in the cerebellum. Isometric head rotation to the left evoked bilateral activation of M1, SMA, anterior and mid-insula, frontal and parietal operculum, putamen, globus pallidus, and S1. In addition, there was significant activation of the ipsilateral caudate, ventrolateral thalamus, middle occipital gyrus, contralateral pre-SMA, and caudal cingulate gyrus. Cerebellar activation was ipsilateral (lobules IV, V, VI, Crus I, and dentate nucleus) with some involvement of the adjacent vermis (vermal lobules V and VI).

The BOLD signal curves for each condition (Fig. 6) suggested that putaminal activation was more prominent ipsilaterally than contralaterally during head rotation to either side. The BOLD curves also showed that ipsilateral lobules VI and Crus I demonstrated specificity for isometric head rotation to the left compared with left wrist extension.

Although we cannot rule out potential hemispheric asymmetries for isometric right or left head rotations, some of the differences observed between these directions may have been due to statistical thresholding effects. Similar to results noted above for isometric hand tasks, relaxing statistical stringency by eliminating the corrections for multiple comparisons revealed bilateral activation of the precentral gyrus and the cerebellum, rather than unilateral activation of these regions.

\section{Discussion}

These results demonstrate that isometric wrist extension activates similar brain regions as prior studies have shown for actual hand movements. Therefore, a similar strategy may reveal brain regions involved with head movements. Isometric head rotation elicited bilateral activation in the precentral gyrus, SMA, insula, frontal operculum, and putamen, as well as ipsilateral activation in the thalamus and cerebellum. Our findings clarify some of the conflicting results obtained previously regarding the hemisphere controlling head movements and the location controlling neck muscles in the motor homunculus.

\section{Is $\mathrm{M} 1$ control of right/left head movements ipsilateral, contralateral or bilateral?}

The hemisphere controlling head movements has been debated extensively, with previous studies providing evidence for ipsilateral, contralateral, or bilateral control (Table 1). These differences may reflect the different methods used. For example, nearly all studies suggesting ipsilateral control were based on the assessment of neck muscle weakness after stroke. In contrast, most studies suggesting bilateral or contralateral control used electrical or magnetic stimulation in healthy individuals. Moreover, most 

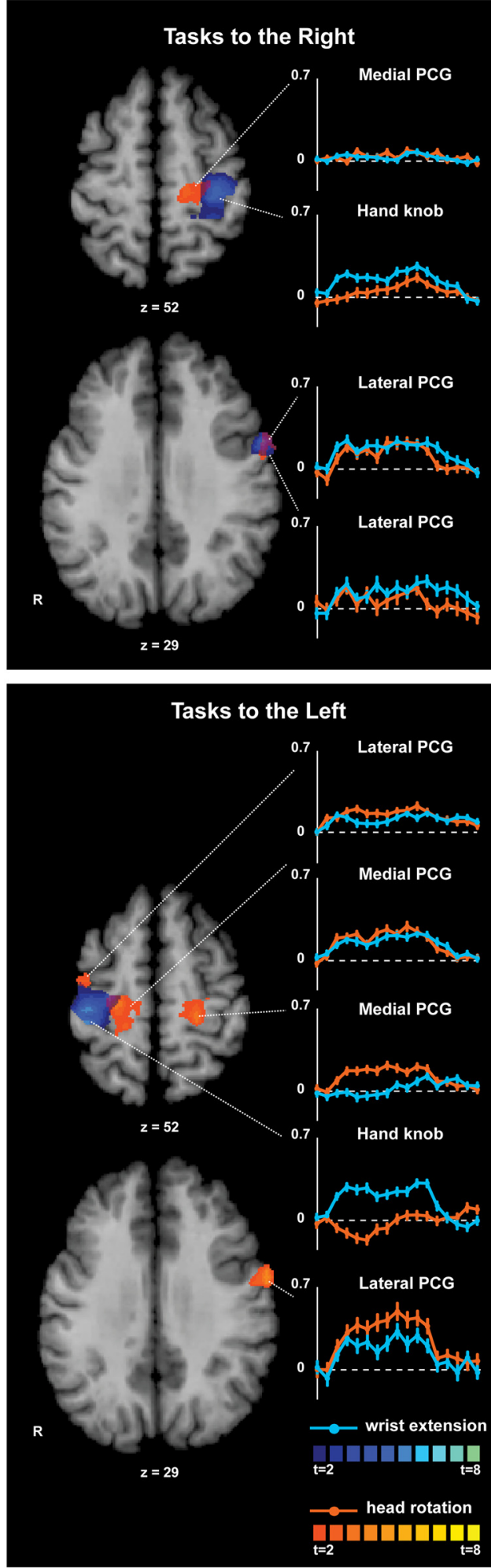

Figure 5. ROI analysis of the precentral gyrus for isometric hand and head tasks compared with rest. Activation maps for hand and head tasks to the same side were superimposed to allow comparisons of the anatomical distribution of activation patterns. Areas with significant activation are shown in blue for hand tasks and in orange for head tasks ( $p<0.05$, corrected for multiple comparisons). Color $t$-scales for each condition are shown at the bottom. BOLD signal curves for each focus of activation show the average percent signal change over time ( $y$-axis: \% $B O L D$ response; $x$-axis: time measured in scans, from -1 to 15 scans). The dashed line shown in each curve represents $0 \%$ or baseline level for the BOLD response. PCG, Precentral gyrus.
Table 3. Coordinates, maximum $t$-values $\left(t_{\max }\right), p$ values, and cluster size for the region of interest analysis

\begin{tabular}{|c|c|c|c|c|c|c|c|c|}
\hline Isometric task & Region & Hemisphere & $x$ & $y$ & $Z$ & $t_{\max }$ & $p$ & Cluster \\
\hline \multirow{2}{*}{$\begin{array}{l}\text { Wrist extension, } \\
\text { right }\end{array}$} & PCG, hand knob & L & -27 & -28 & 49 & 5.32 & 0.00 & 6497 \\
\hline & PCG, lateral/ventral & L & -54 & 5 & 31 & 4.90 & 0.00 & 1405 \\
\hline $\begin{array}{l}\text { Wrist extension, } \\
\text { left }\end{array}$ & $\mathrm{PCG}$, hand knob & $\mathrm{R}$ & 36 & -25 & 49 & 6.17 & 0.00 & 6500 \\
\hline \multirow{2}{*}{$\begin{array}{l}\text { Head rotation, } \\
\text { right }\end{array}$} & $P C G, n$ & L & -15 & -22 & 49 & 3.42 & 0.00 & 1836 \\
\hline & PCG, lateral/ver & L & -54 & -1 & 31 & 3.69 & 0.00 & 730 \\
\hline \multirow{4}{*}{$\begin{array}{l}\text { Head rotation, } \\
\text { left }\end{array}$} & PCG, medial & $\mathrm{R}$ & 21 & -22 & 52 & 3.83 & 0.00 & 2801 \\
\hline & PCG, medial & L & -21 & -28 & 52 & 3.94 & 0.00 & 1714 \\
\hline & PCG, lateral/ & $\mathrm{R}$ & 30 & -13 & 46 & 3.57 & 0.00 & 983 \\
\hline & $\mathrm{PCG}$, lateral/ventral & L & -57 & 2 & 34 & 5.76 & 0.00 & 1836 \\
\hline
\end{tabular}

Significant activation foci with Talairach coordinates (Talairach and Tournoux, 1988).

$P C G$, Precentral gyrus.

studies focused on the role of the contralateral sternocleidomastoid in horizontal head rotation, ignoring important synergistic contributions from the ipsilateral splenius capitis (Vasavada et al., 1998).

We found bilateral precentral gyrus activation during isometric head rotations. These findings suggest that head movements are controlled bilaterally, although contralateral control may be more prominent, as proposed by others (Gandevia and Applegate, 1988; Berardelli et al., 1991; Thompson et al., 1997). Our findings also are consistent with reports that $10-15 \%$ of corticospinal tract fibers projecting to motor neurons are uncrossed in humans (Lemon, 2008) and that these uncrossed pathways can have physiologically meaningful functions (Tazoe and Perez, 2014).

\section{Is the neck area medial or lateral?}

Another basic question has been the location of the neck motor area within the precentral gyrus. Some studies have suggested that this area lies in a region medial to the representation of the upper limb (Obrador, 1953; Thompson et al., 1997; Kang et al., 2011; Pirio Richardson, 2014), whereas others placed it lateral to the hand representation (Rasmussen and Penfield, 1948; Penfield and Rasmussen, 1950). These latter studies mapped somatosensory areas to parallel locations in the motor homunculus, with the sole exception of the neck.

In the present study, two foci in the precentral gyrus of each hemisphere showed activation during isometric head rotation. In both hemispheres, one focus was located medial to the hand knob, whereas the other was more lateral and ventral. These areas do not correspond precisely with the Penfield homunculus (Rasmussen and Penfield, 1948), in which the site of stimulation for head rotation was in the anterior half of the precentral gyrus and lateral to the hand area. There are some possible explanations for these differences. First, in the Penfield studies, the head was restrained by towels, so subtle movements may have been missed. Second, the results for head movements were obtained from only nine patients and were not consistent. For comparison, the results involving limb movements were derived from $>200$ patients. Finally, the neck area in the motor homunculus in the Penfield studies was not determined from rotational movements as in the current study, but instead represented head flexion or retraction or contraction of the trapezius.

Medial and lateral areas in each hemisphere also were identified in a transcranial magnetic stimulation study of the cortical representation of the sternocleidomastoid (Thompson et al., 1997). The investigators suggested that the medial head area represented the sternocleidomastoid, whereas the lateral area repre- 

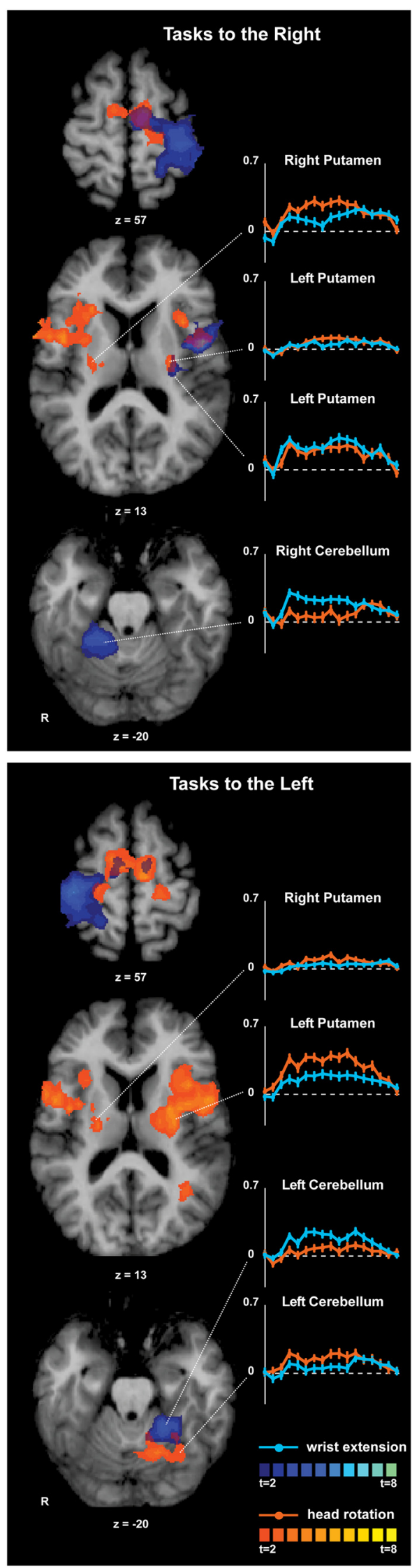

Figure 6. Whole-brain analysis for isometric hand and head tasks compared with rest. Activation maps for hand and head tasks to the same side were superimposed to allow comparisons of the anatomical distribution of activation patterns. Areas with significant activation are
Table 4. Coordinates, maximum $t$-values $\left(t_{\max }\right)$, and $p$ values for whole-brain analyses of hand tasks versus baseline

\begin{tabular}{llcrrrrr}
\hline Isometric task & Region & Hemisphere & \multicolumn{1}{c}{$y$} & \multicolumn{1}{c}{$z$} & $t_{\max }$ & $p$ \\
\hline \multirow{2}{*}{$\begin{array}{c}\text { Wrist extension } \\
\text { right }\end{array}$} & SMA & $\mathrm{L}$ & -6 & -13 & 52 & 7.02 & 0.00 \\
& PMd & $\mathrm{L}$ & -21 & -19 & 64 & 6.07 & 0.00 \\
& PMv & $\mathrm{L}$ & -54 & 5 & 22 & 5.75 & 0.00 \\
& S1 & $\mathrm{L}$ & -33 & -28 & 46 & 5.68 & 0.00 \\
& Parietal operculum & $\mathrm{L}$ & -60 & -34 & 34 & 5.78 & 0.00 \\
& Frontal operculum & $\mathrm{L}$ & -45 & -1 & 13 & 5.73 & 0.00 \\
& Putamen & $\mathrm{L}$ & -27 & -10 & 4 & 4.25 & 0.00 \\
\multirow{5}{*}{ Wrist extension, } & Cerebellum, lobule IV & $\mathrm{R}$ & 9 & -43 & -14 & 5.12 & 0.00 \\
left & SMA & $\mathrm{R}$ & 27 & -40 & -23 & 5.76 & 0.00 \\
& SMA & $\mathrm{L}$ & -9 & -10 & 55 & 3.18 & 0.01 \\
& SMA, superior & $\mathrm{R}$ & 3 & -10 & 46 & 4.36 & 0.00 \\
& Postcentral gyrus & $\mathrm{R}$ & 6 & -13 & 64 & 3.43 & 0.00 \\
& Parietal operculum & $\mathrm{R}$ & 36 & -31 & 49 & 5.99 & 0.00 \\
& Cerebellum, lobule IV-V & $\mathrm{R}$ & 51 & -22 & 37 & 4.60 & 0.00 \\
& Cerebellum, lobule V & $\mathrm{L}$ & -9 & -46 & -14 & 4.08 & 0.00 \\
& Cerebellum, lobule V-VI & $\mathrm{L}$ & -18 & -43 & -17 & 4.46 & 0.00 \\
& & -27 & -40 & -23 & 4.53 & 0.00 \\
\hline
\end{tabular}

Significant activation foci with Talairach coordinates (Talairach and Tournoux, 1988).

sented the platysma. Although we did not monitor the activity of the platysma, this muscle is not typically involved in head movements and therefore this explanation may not be applicable here. However, it remains possible that the medial and lateral precentral foci may control different sets of muscles. Activation of our lateral precentral focus occurred during head rotation to both sides and during right wrist extension. This could be due to movements required to stabilize the body during isometric tasks. Alternatively, the lateral focus may be involved in any isometric motor task or in performing movements after sensory cues. Activation of the inferior precentral gyrus, extending to the frontal operculum and lateral insula, a region similar to that in the present study, was reported in a study of isometric finger movements (van Duinen et al., 2008). The investigators proposed that this area is involved in monitoring feedback and guiding motor performance rather than being directly responsible for finger movements. Therefore, the lateral precentral focus may be involved in any isometric task, whereas the medial precentral focus may have a more prominent role in generating head movements.

The precise anatomical boundaries for M1 proper, PMd, and PMv in humans are only partly delineated (Picard and Strick, 2001; Rizzolatti et al., 2002; Mayka et al., 2006). Therefore, it is not clear whether the medial precentral area identified here in head tasks represents M1 or lies within PMd. Similarly, it is not clear whether the lateral precentral focus in the left hemisphere activated during isometric head rotation to both sides and during right wrist extension should be considered as part of M1 or PMv. The lateral precentral focus in the right hemisphere most likely belongs to PMv because of its location on the precentral sulcus. Considering that M1, PMd, and PMv have direct projections to the spinal cord, all three areas could potentially control neck muscles directly. Regardless of the precise areas activated, the results suggest that both medial and lateral precentral foci are involved in isometric head rotation.

shown in blue for hand tasks and in orange for head tasks $(p<0.05$, corrected for multiple comparisons). Color $t$-scales for each condition are shown on the right. BOLD signal curves from selected sites show the average percent signal change of the BOLD response over time ( $y$-axis: $\%$ BOLD change; $x$-axis: time measured in scans, from -1 to 15 scans). The dashed line shown in each curve represents $0 \%$ or baseline level for the BOLD response. 
Table 5. Coordinates, maximum $t$-values $\left(t_{\max }\right)$, and $p$ values for whole-brain analyses of head tasks versus baseline

\begin{tabular}{|c|c|c|c|c|c|c|c|}
\hline Isometric task & Region & Hemisphere & $x$ & $y$ & $z$ & $t_{\max }$ & $p$ \\
\hline \multirow{13}{*}{$\begin{array}{l}\text { Head rotation, } \\
\text { right }\end{array}$} & SMA & $\mathrm{L}$ & -6 & -13 & 52 & 4.09 & 0.00 \\
\hline & SMA & $\mathrm{R}$ & 9 & -7 & 55 & 3.12 & 0.01 \\
\hline & Anterior insula & L & -33 & 11 & 13 & 4.16 & 0.00 \\
\hline & Anterior insula & $\mathrm{R}$ & 24 & 20 & 13 & 4.12 & 0.00 \\
\hline & Mid-insula & $\mathrm{R}$ & 36 & -1 & 7 & 3.65 & 0.00 \\
\hline & Frontal operculum & $\mathrm{L}$ & -39 & 8 & 7 & 4.32 & 0.00 \\
\hline & Frontal operculum & $\mathrm{R}$ & 42 & -1 & 13 & 4.72 & 0.00 \\
\hline & Putamen, anterior & $\mathrm{R}$ & 27 & -1 & 7 & 4.24 & 0.00 \\
\hline & Putamen, posterior & $\mathrm{L}$ & -27 & -16 & 13 & 3.41 & 0.00 \\
\hline & Putamen, posterior & $\mathrm{R}$ & 24 & -10 & 7 & 4.94 & 0.00 \\
\hline & Globus pallidus & $\mathrm{L}$ & 18 & -6 & 4 & 3.46 & 0.00 \\
\hline & Thalamus, VL, superior & $\mathrm{L}$ & 15 & -10 & 4 & 3.44 & 0.00 \\
\hline & Thalamus, VL, inferior & $\mathrm{L}$ & 18 & -16 & 13 & 2.47 & 0.03 \\
\hline \multirow{27}{*}{$\begin{array}{l}\text { Head rotation, } \\
\text { left }\end{array}$} & SMA & $\mathrm{L}$ & -9 & -13 & 55 & 5.07 & 0.00 \\
\hline & SMA & $\mathrm{R}$ & 9 & -13 & 61 & 4.47 & 0.00 \\
\hline & $\begin{array}{l}\text { Pre-SMA/cingulate gyrus } \\
\text { junction }\end{array}$ & $\mathrm{R}$ & 8 & -1 & 40 & 2.89 & 0.01 \\
\hline & Cingulate sulcus & $\mathrm{L}$ & -18 & 11 & 34 & 2.97 & 0.01 \\
\hline & Posterior cingulate sulcus & $\mathrm{R}$ & 21 & -34 & 31 & 2.80 & 0.01 \\
\hline & S1 & $\mathrm{R}$ & 60 & -19 & 31 & 3.61 & 0.00 \\
\hline & Parietal operculum & $\mathrm{R}$ & 57 & -31 & 31 & 3.98 & 0.00 \\
\hline & S1/parietal operculum & $\mathrm{L}$ & -61 & -34 & 34 & 5.02 & 0.00 \\
\hline & S1/parietal operculum & $\mathrm{L}$ & -57 & -28 & 25 & 4.79 & 0.00 \\
\hline & $\begin{array}{l}\text { Superior temporal sulcus/ } \\
\text { MOG }\end{array}$ & $\mathrm{L}$ & -30 & -55 & 19 & 3.79 & 0.00 \\
\hline & Frontal operculum & $\mathrm{L}$ & -48 & 2 & 13 & 4.83 & 0.00 \\
\hline & Frontal operculum & $\mathrm{R}$ & 39 & -4 & 13 & 4.64 & 0.00 \\
\hline & Anterior insula & $\mathrm{L}$ & -36 & 11 & 7 & 6.31 & 0.00 \\
\hline & Anterior insula & $\mathrm{R}$ & 30 & 14 & 10 & 2.97 & 0.01 \\
\hline & Mid-insula & $\mathrm{L}$ & -39 & -1 & 4 & 6.49 & 0.00 \\
\hline & Mid-insula & $\mathrm{R}$ & 33 & -1 & 10 & 3.55 & 0.00 \\
\hline & Caudate head & $\mathrm{L}$ & -15 & -1 & 31 & 4.20 & 0.00 \\
\hline & Thalamus, VL & $\mathrm{L}$ & -15 & -13 & 4 & 4.28 & 0.00 \\
\hline & Putamen, mid & $\mathrm{L}$ & -27 & -13 & 10 & 4.94 & 0.00 \\
\hline & Putamen, mid & $\mathrm{R}$ & 24 & -4 & 13 & 2.79 & 0.01 \\
\hline & Putamen, posterior & $\mathrm{L}$ & -27 & -10 & 4 & 6.26 & 0.00 \\
\hline & Putamen, superior & $\mathrm{R}$ & 24 & -10 & 16 & 4.40 & 0.00 \\
\hline & Globus pallidus & $\mathrm{L}$ & -18 & -10 & 7 & 4.08 & 0.00 \\
\hline & Globus pallidus & $\mathrm{R}$ & 21 & -14 & 13 & 3.57 & 0.00 \\
\hline & Cerebellum, lobule VI & $\mathrm{L}$ & -15 & -61 & -17 & 4.58 & 0.00 \\
\hline & Cerebellum, Crus I & $\mathrm{L}$ & -36 & -49 & -39 & 3.92 & 0.00 \\
\hline & Dentate nucleus & L & -18 & -34 & -32 & 3.32 & 0.00 \\
\hline
\end{tabular}

Significant activation foci with Talairach coordinates (Talairach and Tournoux, 1988).

MOG, Middle occipital gyrus; VL, ventrolateral nucleus.

\section{Role of other brain regions}

Animal studies have shown that head movements can be elicited by experimental manipulations of many regions, including the cerebral cortex, cerebellum, basal ganglia, and several midbrain and brainstem regions (Fukushima, 1987; Isa and Sasaki, 2002). Similar evidence comes from human studies showing that lesions in many regions can cause abnormal movements of the head (LeDoux and Brady, 2003).

The basal ganglia are thought to have a leg-arm-face somatotopic organization (Scholz et al., 2000; Gerardin et al., 2003; Nambu, 2011), but data concerning the neck are limited. Our findings revealed bilateral activation of the putamen (with ipsilateral predominance) during isometric head rotations, with activation more dorsal than for hand tasks. Bilateral basal ganglia activation similarly has been reported in prior studies of movements of the hands and other body parts even when M1 activation is unilateral (Scholz et al., 2000; Gerardin et al., 2003).
Historically, the cerebellar hemispheres were viewed as being involved in the control of limb movements, whereas the vermis was associated with control of axial muscles (Manni and Petrosini, 2004; Mottolese et al., 2013). However, more recent studies have suggested that the cerebellum has a fractured somatotopic organization. We observed activation of the ipsilateral cerebellar hemisphere and vermis during head tasks that overlapped with areas activated during hand tasks. The relatively broad activations of the cerebellum may reflect its fractured somatotopic organization and do not support the view of the hemispheres as being exclusively responsible for the control of limb movements.

\section{Limitations and future studies}

Our study had some shortcomings that should be acknowledged. The main limitation is that we cannot link specific regions of brain activation with specific muscles responsible for head rotation. As noted above, rotating the head to one side involves activation of the contralateral sternocleidomastoid and ipsilateral splenius capitis. It is therefore feasible that activating both muscles on opposite sides of the body is reflected by bilateral activation of the precentral gyrus, even though control of individual muscles could be strictly contralateral. It also is feasible that the medial and lateral locations identified in the precentral gyrus each reflect activation of one of these muscles. These possibilities seem unlikely because transcranial magnetic stimulation studies have implied that the sternocleidomastoid has bilateral representation in the precentral gyrus, whereas the splenius capitis has either contralateral or bilateral representation (Benecke et al., 1988; Gandevia and Applegate, 1988; Berardelli et al., 1991; Thompson et al., 1997).

Another limitation of our fMRI study is its limited spatial resolution, which does not allow reliable identification of small subcortical regions. A related technical limitation is that complete coverage of the most caudal portions of the brainstem and cerebellum was not feasible, so no conclusions can be made about those regions. In addition, our protocol did not allow dissociation of motor signals from proprioceptive signals during isometric tasks. This caveat is relevant to all studies of motor control because it is challenging to eliminate somatosensory signals generated from movement itself. Additionally, our study was focused on horizontal head rotation, so the findings may not be applicable to head movements in other directions. Finally, our study could not verify whether isometric head rotations evoke similar patterns of brain activation as actual head movements.

\section{Conclusions}

These findings provide new information regarding the neural control of head movements in humans. Our results suggest that isometric tasks may provide a suitable method for investigating head movements, bypassing some of the normal limitations of brain imaging. The current results suggest that head movements in humans are controlled bilaterally in the precentral gyrus, but with contralateral predominance. The results also provide evidence for regions within the cerebellum and basal ganglia in controlling head movements. Future studies may use similar methods to investigate head movements in other directions, as well as disorders affecting head motor control such as tremor and cervical dystonia. 


\section{References}

Adamo DE, Scotland S, Martin BJ (2012) Upper limb kinesthetic asymmetries: gender and handedness effects. Neurosci Lett 516:188-192. CrossRef Medline

Anagnostou E, Paraskevas GP, Spengos K, Vassilopoulou S, Zis V, Vassilopoulos D (2011) Same or opposite? Association of head-movement weakness with limb paresis in stroke. Neurologist 17:309-311. CrossRef Medline

Balagura S, Katz RG (1980) Undecussated innervation to the sternocleidomastoid muscle: a reinstatement. Ann Neurol 7:84-85. CrossRef Medline

Beevor CE (1909) Remarks on the paralysis of the movements of the trunk in hemiplegia, and the muscles which are affected. Br Med J 1:881-885. CrossRef Medline

Benecke R, Meyer BU, Schönle P, Conrad B (1988) Transcranial magnetic stimulation of the human brain: responses in muscles supplied by cranial nerves. Exp Brain Res 71:623-632. CrossRef Medline

Berardelli A, Priori A, Inghilleri M, Cruccu G, Mercuri B, Manfredi M (1991) Corticobulbar and corticospinal projections to neck muscle motoneurons in man: a functional study with magnetic and electric transcranial brain stimulation. Exp Brain Res 87:402-406. Medline

Cho ZH (2010) 7.0 Tesla MRI brain atlas: in vivo atlas with cryomacrotome correlation, Ed 1. New York: Springer.

Criswell E, Cram R (2010) Cram's introduction to surface electromyography, Ed 2. New York: Jones and Bartlett Publishers.

DeToledo JC, Dow R (1998) Sternomastoid function during hemispheric suppression by amytal: insights into the inputs to the spinal accessory nerve nucleus. Mov Disord 13:809-812. CrossRef Medline

Divekar NV, John LR (2013) Neurophysiological, behavioural and perceptual differences between wrist flexion and extension related to sensorimotor monitoring as shown by corticomuscular coherence. Clin Neurophysiol 124:136-147. CrossRef Medline

Duvernoy HM (1999) The human brain. Surface, blood supply and threedimensional sectional anatomy, Ed 2. New York: Springer.

Forman SD, Cohen JD, Fitzgerald M, Eddy WF, Mintun MA, Noll DC (1995) Improved assessment of significant activation in functional magnetic resonance imaging (fMRI): use of a cluster-size threshold. Magn Reson Med 33:636-647. CrossRef Medline

Fukushima K (1987) The interstitial nucleus of Cajal and its role in the control of movements of head and eyes. Prog Neurobiol 29:107-192. CrossRef Medline

Gandevia SC, Applegate C (1988) Activation of neck muscles from the human motor cortex. Brain 111:801-813. CrossRef Medline

Gerardin E, Lehéricy S, Pochon JB, Tézenas du Montcel S, Mangin JF, Poupon F, Agid Y, Le Bihan D, Marsault C (2003) Foot, hand, face and eye representation in the human striatum. Cereb Cortex 13:162-169. CrossRef Medline

Goebel R, Esposito F, Formisano E (2006) Analysis of functional image analysis contest (FIAC) data with brainvoyager QX: From single-subject to cortically aligned group general linear model analysis and selforganizing group independent component analysis. Hum Brain Mapp 27:392-401. CrossRef Medline

Hanajima R, Ugawa Y, Terao Y, Sakai K, Furubayashi T, Machii K, Uesugi H, Mochizuki H, Kanazawa I (1998) Cortico-cortical inhibition of the motor cortical area projecting to sternocleidomastoid muscle in normals and patients with spasmodic torticollis or essential tremor. Electroencephalogr Clin Neurophysiol 109:391-396. CrossRef Medline

Isa T, Sasaki S (2002) Brainstem control of head movements during orienting; organization of the premotor circuits. Prog Neurobiol 66:205-241. CrossRef Medline

Kang SY, Ma HI, Lee MJ, Kwon SB, Jung S, Kim YJ, Hwang SH (2011) Ipsilateral tilt and contralateral sensory change of neck in cortical infarction. J Clin Neurol 7:156-158. CrossRef Medline

Keisker B, Hepp-Reymond MC, Blickenstorfer A, Kollias SS (2010) Differential representation of dynamic and static power grip force in the sensorimotor network. Eur J Neurosci 31:1483-1491. CrossRef Medline

LeDoux MS, Brady KA (2003) Secondary cervical dystonia associated with structural lesions of the central nervous system. Mov Disord 18:60-69. CrossRef Medline

Lemon RN (2008) Descending pathways in motor control. Annu Rev Neurosci 31:195-218. CrossRef Medline
Manni E, Petrosini L (2004) A century of cerebellar somatotopy: a debated representation. Nat Rev Neurosci 5:241-249. Medline

Manon-Espaillat R, Ruff RL (1988) Dissociated weakness of sternocleidomastoid and trapezius muscles with lesions in the CNS. Neurology 38 : 796-797. CrossRef Medline

Mastaglia FL, Knezevic W, Thompson PD (1986) Weakness of head turning in hemiplegia: a quantitative study. J Neurol Neurosurg Psychiatry 49: 195-197. CrossRef Medline

Mayka MA, Corcos DM, Leurgans SE, Vaillancourt DE (2006) Threedimensional locations and boundaries of motor and premotor cortices as defined by functional brain imaging: a meta-analysis. Neuroimage 31: 1453-1474. CrossRef Medline

Mottolese C, Richard N, Harquel S, Szathmari A, Sirigu A, Desmurget M (2013) Mapping motor representations in the human cerebellum. Brain 136:330-342. CrossRef Medline

Nambu A (2011) Somatotopic organization of the primate basal ganglia. Front Neuroanat 5:26. Medline

Nöth U, Laufs H, Stoermer R, Deichmann R (2012) Simultaneous electroencephalography-functional MRI at $3 \mathrm{~T}$ : an analysis of safety risks imposed by performing anatomical reference scans with the EEG equipment in place. J Magn Reson Imaging 35:561-571. CrossRef Medline

Obrador S (1953) Clinical observations on the representation of the neck movements within the primary motor cortex. Electroencephalogr Clin Neurophysiol 5:611-612. CrossRef Medline

Odergren T, Rimpiläinen I (1996) Activation and suppression of the sternocleidomastoid muscle induced by transcranial magnetic stimulation. Electroencephalogr Clin Neurophysiol 101:175-180. CrossRef Medline

Odergren T, Rimpiläinen I, Borg J (1997) Sternocleidomastoid muscle responses to transcranial magnetic stimulation in patients with cervical dystonia. Electroencephalogr Clin Neurophysiol 105:44-52. CrossRef Medline

Penfield W, Rasmussen T (1950) The cerebral cortex of man: a clinical study of localization of function. New York: Macmillan.

Peterson BW (2004) Current approaches and future directions to understanding control of head movement. Prog Brain Res 143:369-381. Medline

Picard N, Strick PL (2001) Imaging the premotor areas. Curr Opin Neurobiol 11:663-672. CrossRef Medline

Pirio Richardson S (2014) Enhanced dorsal premotor-motor inhibition in cervical dystonia. Clin Neurophysiol. In press.

Poldrack RA, Mumford JA, Nichols TE (2011) Handbook of functional MRI data analysis, Ed 1. New York: Cambridge University.

Rasmussen T, Penfield W (1948) Movement of head and eyes from stimulation of human frontal cortex. Res Publ Assoc Res Nerv Ment Dis 27: 346-361. Medline

Rizzolatti G, Fogassi L, Gallese V (2002) Motor and cognitive functions of the ventral premotor cortex. Curr Opin Neurobiol 12:149-154. CrossRef Medline

Sacher J, Neumann J, Okon-Singer H, Gotowiec S, Villringer A (2013) Sexual dimorphism in the human brain: evidence from neuroimaging. Magn Reson Imaging 31:366-375. CrossRef Medline

Schmahmann JD, Doyon J, McDonald D, Holmes C, Lavoie K, Hurwitz AS, Kabani N, Toga A, Evans A, Petrides M (1999) Three-dimensional MRI atlas of the human cerebellum in proportional stereotaxic space. Neuroimage 10:233-260. CrossRef Medline

Scholz VH, Flaherty AW, Kraft E, Keltner JR, Kwong KK, Chen YI, Rosen BR, Jenkins BG (2000) Laterality, somatotopy and reproducibility of the basal ganglia and motor cortex during motor tasks. Brain Res 879:204215. CrossRef Medline

Talairach J, Tournoux P (1988) Co-planar stereotaxic atlas of the human brain: 3-dimensional proportional system: an approach to cerebral imaging. New York: Thieme Medical Publishers.

Tazoe T, Perez MA (2014) Selective activation of ipsilateral motor pathways in intact humans. J Neurosci 34:13924-13934. CrossRef Medline

Thesen S, Heid O, Mueller E, Schad LR (2000) Prospective acquisition correction for head motion with image-based tracking for real-time fMRI. Magn Reson Med 44:457-465. Medline

Thompson ML, Thickbroom GW, Mastaglia FL (1997) Corticomotor 
representation of the sternocleidomastoid muscle. Brain 120:245-255. CrossRef Medline

Triggs WJ, Subramanium B, Rossi F (1999) Hand preference and transcranial magnetic stimulation asymmetry of cortical motor representation. Brain Res 835:324-329. CrossRef Medline

van Duinen H, Renken R, Maurits NM, Zijdewind I (2008) Relation between muscle and brain activity during isometric contractions of the first dorsal interosseus muscle. Hum Brain Mapp 29:281-299. CrossRef Medline

Vasavada AN, Li S, Delp SL (1998) Influence of muscle morphometry and moment arms on the moment-generating capacity of human neck muscles. Spine 23:412-422. CrossRef Medline
Vitti M, Fujiwara M, Basmanjian JM, Iida M (1973) The integrated roles of longus colli and sternocleidomastoid muscles: an electromyographic study. Anat Rec 177:471-484. CrossRef Medline

White T, O'Leary D, Magnotta V, Arndt S, Flaum M, Andreasen NC (2001) Anatomic and functional variability: the effects of filter size in group fMRI data analysis. Neuroimage 13:577-588. Medline

Willoughby EW, Anderson NE (1984) Lower cranial nerve motor function in unilateral vascular lesions of the cerebral hemisphere. Br Med J 289: 791-794. CrossRef Medline

Yousry TA, Schmid UD, Alkadhi H, Schmidt D, Peraud A, Buettner A, Winkler P (1997) Localization of the motor hand area to a knob on the precentral gyrus: a new landmark. Brain 120:141-157. CrossRef Medline 